\title{
Food avoidance behaviour among children aged 2-6 years in North Jakarta and its correlation with weight and energy intake
}

Received 22 October 2020 Accepted 18 September 2020

Link to DOI:

10.25220/WNJ.V05.i1.0016

Journal Website: www.worldnutrijournal.org

\author{
Alfi Rahma Putri, ${ }^{1}$ Dian Novita Chandra, ${ }^{1}$ Luh Ade Ari Wiradnyani ${ }^{2}$ \\ 1. Department of Nutrition, Faculty of Medicine, Universitas Indonesia, Dr. Cipto Mangunkusumo \\ Hospital, Jakarta, Indonesia \\ 2. Southeast Asian Ministers of Education Organization Regional Center for Food and Nutrition \\ (SEAMEO-RECFON) - Pusat Kajian Gizi Regional (PKGR), Universitas Indonesia
}

\begin{abstract}
Background: Children generally facing food avoidance or food refusal behaviour and it may affect their food consumption. But there is limited information on food avoidance behaviour among Indonesian children. Therefore, this study aimed to assess whether energy intake and body weight are influenced by food avoidance behaviour in children aged 2-6 years in North Jakarta.

Methods: Participants ( $N=168)$ were recruited between February - March 2020. Data collection was obtained via interviews with the mother or caregiver of the children. Food avoidance behaviour consisting of satiety responsiveness, slowness in eating, emotional under-eating, and food fussiness was measured with the Child Eating Behaviour Questionnaire. The child's weight and dietary intake were assessed using a digital weighing scale and $2 \times 24$ hours food recall. Spearman test was performed to analyse the correlation between these variables. Multivariate analysis was done using linear regression to determine predictors of a child's body weight and energy intake.

Results: Energy intake among children was below the recommendation, and it was significantly correlated with the child's body weight. Satiety responsiveness was negatively correlated with the child's body weight $(r=-0.166 ; p$-value $<0.05)$ and energy intake $(r=-0.210 ; p$-value $<0.05)$. After running a linear regression test, we found that satiety responsiveness along with family income and child's age was a significant predictor of energy intake among children.

Conclusions: Children who are more responsive to satiety had lower body weight and energy intake. Understanding the child's food avoidance behaviour is useful for designing intervention programs related to optimizing intake in children and malnutrition.
\end{abstract}

Keywords children, food avoidance behaviour, energy intake, body weight

\section{Introduction}

Currently, Indonesia is facing the double burden of malnutrition, where the prevalence of undernutrition remains high, but the prevalence of overweight and obesity at all ages is increasing over time, particularly in DKI Jakarta province. ${ }^{1}$ Malnutrition is linked to other health problems, such as infectious

\section{Corresponding author:}

Alfi Rahma Putri, S.Tr. Gz

Department of Nutrition, Faculty of Medicine, Universitas

Indonesia

Jl. Salemba Raya no.6, Central Jakarta, Indonesia

Email: alfirahmaputri2@gmail.com diseases and developmental disorders in children, and this can lead to an increase in child mortality. One of the factors contributing to this malnutrition problem is dietary intake. Along with infectious diseases, maternal health, feeding practice, dietary intake was a factor that related to wasting, overweight, and stunting among children. ${ }^{2,3}$ In Indonesia, a study from South East Asian Nutrition Survey (SEANUTS I) showed that the nutrient intake of Indonesian children was below the Indonesian Recommended Daily Allowance (RDA), and the major problem among Indonesian children was stunting and underweight. ${ }^{4}$ The results of the 
SEANUTS study in Malaysia also showed that 30\% of the children there could not meet their energy requirement, but the prevalence of overnutrition was still higher than undernutrition in Malaysian children. $^{5}$

Children's diet is influenced by multiple factors, including mother's education, mother occupation, family income, parental feeding practice, and children eating behaviour. ${ }^{6,7}$ Studies showed that mother nutrition knowledge and occupation were related to dietary adequacy among children because a mother have a big influence on determining what, when, and how much food is provided to their children directly. ${ }^{8,9}$ In addition, family income was related to the family's ability to access food. ${ }^{10}$ Parental feeding practices have a bidirectional relationship with child eating behaviour. The practices can influence and/or respond to child eating behaviours, which in turn affects the child's food intake. For instance, the pressure to eat practice was associated with increased food avoidance and decreasing preferences on the consumption of the tobe eaten foods. ${ }^{11,12}$ While responsive feeding including modelling healthy eating and involved with children during meals can contribute to healthy eating behaviour as well as promote optimal food intake among children. ${ }^{13}$

In this study, we try to focus on children eating behaviour, particularly food avoidance behaviour, that may influence dietary intake and diet quality. Food avoidance behaviour refers to the child's tendency to avoid or reject certain foods. Children generally facing food avoidance or food refusal behaviour, especially children aged 2-6 years and this behaviour was associated with lower fruit and vegetable intake and less dietary variety. ${ }^{14}$ The Child Eating Behaviour Questionnaire (CEBQ) was used to measure food avoidance behaviour. Food avoidance behaviour consists of four scales, namely satiety responsiveness, slowness in eating, emotional under-eating, and food fussiness. A high score on food avoidance behaviour indicates a high tendency to avoid or refuse foods. ${ }^{15}$

The relationship between the food avoidance scale and children's diet is often stated. Previous studies showed that higher score on food avoidance behaviour was associated with lower meal size and higher intake of snack food and less intake of fruits and vegetables. ${ }^{7,16,17}$ Moreover, a study among school children in the UK reported that satiety responsiveness, slowness in eating, and food fussiness was negatively associated with child weight. ${ }^{18}$ This is supported by findings in children under-five in Australia, where satiety responsiveness and slowness in eating were inversely correlated with energy intake of lunch meal and child's BMI. ${ }^{19}$ Thus, assessing food avoidance behaviour in children may help identify children who are at high risk of becoming obese or underweight. To our knowledge, a study on eating behaviour especially for food avoidance scale in Indonesian children is still limited. Then, exploring the association between food avoidance with dietary intake and body weight might contribute to strengthening programs or interventions to prevent nutritional problems among children. This study aimed to assess whether energy intake and body weight are influenced by food avoidance behaviour in children aged 2-6 years in North Jakarta.

\section{Methods}

\section{Study design}

The study is a cross-sectional study. Data collection of this study was done from February until March 2020 in Jakarta Province. Jakarta, as one of the most densely populated provinces with many slum areas, was chosen purposively. Then, through multistage random sampling, Pejagalan village located in North Jakarta was selected as the study area.

\section{Subjects}

In Pejagalan village, four child health care posyandu was randomly selected. Posyandu is a communitybased integrated health post providing some basic health and nutrition services mainly for young children and pregnant women, such as growth monitoring, nutrition supplementation, immunization, and antenatal care. Using the list of children in posyandu and family registered certificate (kartu keluarga) in the selected posyandu area, consecutive sampling was carried out to select subjects who meet the inclusion criteria. The inclusion criteria for subject selection were male or female children aged $2.0-6.9$ years and were healthy, the mother agreed to sign informed consent 
and were able to communicate in Bahasa Indonesia. While, mothers who report that their children had a food allergy or food intolerance were excluded from this study. For bivariate analysis, the sample size was calculated by using the formula of correlation estimation for cross-sectional survey, and for multivariate analysis sample size was calculate based on the rule of thumb of 10 subjects for each variable in the model. After calculating the sample size for bivariate and multivariate analysis, a total minimum sample needed for this study was 168 of the children.

\section{Child's weight}

It was measured by a qualified enumerator using SECA 876 digital weighing scale to the nearest 0.1 $\mathrm{kg}$. According to the standardized procedure, children were asked to remove shoes and heavy clothing before the measurement. ${ }^{20}$ Measurement were taken twice, and the third measurement was taken when the difference between the two measurements was more than $0.1 \mathrm{~kg}$.

\section{Questionnaire}

Data collection was obtained via interviews with the mother or caregiver of the children by trained enumerators. The time needed to interview each respondent during the survey was approximately 45-60 minutes. Mother or caregiver reported their sociodemographic information, such as child's birth date, sex, mother education, mother occupation, caregiver relationship, and family income. Mother education was categorized into low education $(\leq 9$ years of schooling, e.g., Junior High School and below) and high education ( $\geq 10$ years of schooling, e.g., Senior High School and above). While mother occupation was categorized into unemployed (housewife), partially employed (a mother who works less than 30 hours in a week), and fully employed (a mother who works at least 35-40 hours in a week or 8 hours in a day). Then, family income was divided into three categories; low income (below the first quartile), middle income (between first to the fourth quartile), and high income (above the fourth quartile).

\section{Child eating behaviour questionnaire}

Food avoidance behaviours were assessed using the CEBQ. In this study, the 15 items questionnaire consist of four food avoidance subscales: satiety responsiveness (5 items; e.g., my child has a big appetite), slowness in eating (3 items; e.g. my child eats slowly), emotional under-eating (3 items; e.g. my child eats less when angry), and food fussiness (4 items; e.g. my child refuses new foods at first). Mothers were asked to rate their children's behaviour related to food avoidance on a 5-point Likert scale ranging from "never" to "always" and each item will be scored 1 (Lowest) to 5 (Highest). The mean score (range $1-5$ ) was calculated for each subscale. Internal reliability (Cronbach alpha) was calculated for each subscale of CEBQ, and after deleting four items on CEBQ, good internal reliability was found in four subscales with values of Cronbach alpha ranging from $0.711-0.751$. The deleted items are "My child takes more than 30 minutes to finish a meal" from slowness in eating scale, "My child eats more when he/she is happy" from emotional under-eating scale, "My child enjoys a wide variety of foods" and "My child is difficult to please with meals" from food fussiness scale. ${ }^{15}$

\section{Dietary assessment}

Children's dietary intake was assessed using 2 x 24 hours food recall. Mothers were asked to recall all foods and beverages consumed by their child during the previous day (00:00-24:00). During the dietary assessment, an error might occur due to memory bias or inaccurate estimation on the portion size and cause misreporting data. In this study, under/overreport energy intake was calculated through the ratio of reported energy intake to BMR. Basal Metabolic Rate was estimated from the Schofield equation, and the cut off for under-, plausible, and overreport energy intake was based on Börnhorst et al. 2012. ${ }^{21}$ Energy intake was categorized into two categories, adequate and risk of inadequate. Adequate energy intake refers to the proportion of subjects that consume amounts of energy above the recommendation. The following formula of EER was used to determine the 
individual energy requirement based on age, sex, body weight and height, and also physical activity level.

\section{Statistical analysis}

Before running the analysis, we excluded cases with incomplete data and under/overreport energy intake. All the collected data obtained from the questionnaire were entered in SPSS software version 20.0, and dietary assessments were entered and transformed into energy intake using NutriSurvey software program. After combining data from energy intake, data analysis was continued with SPSS software to run the univariate, bivariate, and multivariate analysis. Univariate analysis was used to describe socio-demographic and economic characteristics, children's food avoidance behaviour score, child's body weight, and energy intake. Numerical data are presented as mean \pm SD or median (IQR) depend on the distribution of the data and categorical data are presented as a proportion (\%). Normality test was conducted using Kolmogorov test and coefficient of variance. For Kolmogorov test, significance was set at p-value $>$ 0.05 , and for coefficient variance, data was normally distributed if the value was $\leq 20$ percent. Since the food avoidance behaviour data were not normally distributed, we ran Spearman test to assess the correlation between food avoidance score with child's body weight and energy intake. While multivariate analysis was done using linear regression to determine predictors of a child's body weight and energy intake. These analyses were considered significant if the $\mathrm{p}$-value $<0.05$.

\section{Ethical consideration}

This study was conducted after receiving approval from the Ethical Committee of Faculty of Medicines, Universitas Indonesia No. ND6/UN2.F1/ETIK/PPM.00.02/2020. Before data collection, approval was also obtained from the local authority, health district office, and Primary Health Care. During data collection, the mothers independently signed informed consent before the interview.

\section{Results}

The final total sample of this study was 168 children. Table 1. shows the sociodemographic characteristics of the subjects. The majority of the children were boys $(63.1 \%)$. The median of child's age was four years old and there was similar distribution in children aged 3 years old $(22.6 \%)$ and 4 years old $(24.4 \%)$. According to mother education and occupation, $67.3 \%$ of the mother have low education and the majority of the mother were unemployed $(66.7 \%)$. Most of the children were taken care of by their parents $(91.1 \%)$. The median household income was IDR 3.05 million and the majority of children lived in households that had a lower middle income $(76.2 \%)$. Table 1 . also presents the distribution of a child's body weight and energy intake. Children's body weight showed the median of $15.3 \mathrm{~kg}$, and the median of energy intake for all children was $1307.3 \mathrm{kcal}$, and both of these variables were not normally distributed. Energy intake data obtained from multiple food recall 24-hours was categorized as adequate and risk of inadequate. These findings suggest that more than half of our subjects had a risk of inadequate energy intake. Further analysis showed that body weight and energy intake among children have a significant correlation with their age, and a child's body weight also differ significantly by mother's education level. The higher median of body weight was found among children with mother who has low education.

Table 2. shows the distribution of food avoidance behaviour scores among our subjects. From the table, we can see that the median scores for food avoidance scales range from 2.6 to 3.3 . The emotional under-eating scale has the highest median, followed by satiety responsiveness at the second. Further analysis showed that the satiety responsiveness scale had a significant association with the family income, in which the median satiety responsiveness scale was higher among children from higher-income families.

Bivariate analysis was performed to determine the correlation between the food avoidance behaviour scale with a child's weight and energy intake. Table 3 displayed the correlation between these variables using the Spearman test. All of food avoidance scales were positively correlated with one 
another, except the correlation between food fussiness scale with slowness in eating and emotional under-eating. Overall, the result showed that satiety responsiveness had a negative correlation with child's body weight $(\mathrm{r}=-0.166$; $\mathrm{p}$ value $<0.05)$ and energy intake $(r=-0.210 ; p$-value $<0.05)$. Moreover, energy intake also positively correlated with child' body weight $(\mathrm{r}=0.354$; $\mathrm{p}$ value $<0.01)$.

Table 4. displayed the summary of multiple linear regression analysis. A significant predictor of a child's body weight was the child's age $(\beta=1.370$, p-value $<0.01$ ), in which, an increase of one unit on child's age corresponds to an increase of 1.370 units on body weight. While, significant predictors of energy intake were child's age $(\beta=0.715$, $p$-value $<0.05)$, family income $(\beta=0.000, p$-value $<0.01)$ and satiety responsiveness $(\beta=-1.736, p$-value $<0.01)$. For every unit increase in satiety responsiveness score, energy intake among children decreases 1.736 times.

\section{Discussion}

This present study reveals that energy intake among children in Jakarta was below the recommendation. This is similar to the study of SEANUTS I that showed the percentage of Indonesian children who have dietary intake below the recommendation was high $(21 \%-63 \%) .{ }^{4}$ Childhood is a period of rapid growth and development. Nutritional needs during this period increased as the child gets older, and in line with our finding, the older the children the greater their energy intake, and the energy intake significantly correlated with their body weight. For a long time, a child's inadequate body weight can trigger various health problems. ${ }^{2}$ Study among Indonesian pre-school children showed that inadequate energy intake was also related to an increased risk of developmental delay. ${ }^{22}$

This study hypothesizes, there is a correlation between food avoidance behaviour with a child's body weight and energy intake among children aged 2-6 years. In this study, food avoidance related to eating behaviour was represented by satiety responsiveness, slowness in eating, emotional under-eating and food fussiness. Based on the result of our study, the children seem to have an emotional tendency to eat less and be more responsive to satiety. The result also showed that satiety responsiveness had a significant correlation with a child's body weight and energy intake. The higher the score on the satiety responsiveness scale, the lower the child's body weight and energy intake. This finding is in line with previous researches that showed four scales of food avoidance behaviour were negatively correlated with body weight and dietary intake and it was usually related to obesityreducing behaviour, such as lower meal size. ${ }^{7,16}$

In the final regression model, a child's age, family income, and satiety responsiveness were a significant predictor of energy intake and the significant predictor for a child's weight was only found on child's age. Family income was found as a predictor of energy intake. Increasing family income contributes to greater energy intake among children. A previous study showed families with low socioeconomic status tend to have a weaker purchasing power to provide enough food in their home. ${ }^{10}$ They also have difficulty in accessing healthy food. Study in Swedish, healthy eating was associated with higher diet costs due to the different prices between healthy food and unhealthy food. Thus, families with high income are more likely to access adequate and healthy foods. ${ }^{23}$

In this study, satiety responsiveness appeared to be the strongest predictor of a child's energy intake. Satiety responsiveness defines as a child's response to satiety or internal signal to finish eating, and the more responsive the child to satiety the more likely the child to leave their food. ${ }^{24}$ This finding supports the crucial role of parents in improving a child's self-regulation ability, particularly responsiveness to satiety cues. Satiety responsiveness among children is also related to the type of food preference and food consumption. Previous studies have found that children who are more responsive to satiety tend to have a lower preference for fruits and vegetables, ${ }^{25}$ lower consumption of vegetables, cheese, and meat, and higher consumption of energy-dense foods such as, candies and chocolate. ${ }^{26}$ Satiety was related to the diet composition and consuming high-energy foods that were relatively satiating can provide pleasant feeling of fullness. ${ }^{27}$ This is in line with our finding which showed children get full up easily.

Supporting the hypothesis, evidence from the previous studies showed that satiety responsiveness and slowness in eating were related to dietary intake 
among children. ${ }^{19,26}$ In our finding, satiety responsiveness was related to slowness in eating. Slowness in eating refers to the reduction of a child's speed in eating and is caused by a lack of interest and enjoyment of food. The child who has a higher score of slowness in eating scale will be taking a long time to finish their meals. ${ }^{28}$ Previous study expected that the eating rate among children will be getting slow when they are more responsive to satiety. ${ }^{29}$ Children with this behaviour are more likely to have inadequate intake of food, and then it will put them at risk of underweight and undernutrition. ${ }^{26}$ Moreover, several studies have shown a relationship between eating behaviour with child's body weight. Satiety responsiveness and slowness in eating were inversely related to the child's BMI. $7,18,19,30$ On the contrary, our finding shows that slowness in eating was a positive predictor of energy intake, but the association was not significant.

In contrast with our hypothesis and finding from the previous studies, the significant correlation between emotional under-eating and food fussiness with energy intake and body weight were not found in this study. Whereas several studies showed that these behaviours are also related to a child's dietary intake and body weight. ${ }^{7,18}$ Emotional under-eating refers to children who will eat less in response to their emotions, such as angry, tired, happy, upset, etc. ${ }^{28}$ While food fussiness refers to the rejection of unfamiliar and/or familiar food and children with food fussiness are more selective about which foods they want to eat. ${ }^{31}$

Despite our strength by measuring a child's eating behaviour, dietary intake, and body weight in one study, there are several limitations in our study that need to be considered. First, we use the reference height in the Indonesian RDA, not the actual height of each child in categorizing children's energy intake. Second, the CEBQ used in this study was modified from its original version for better reliability. It might impact the interpretation of the food avoidance results of the study. Third, given the characteristics of the subjects, this study cannot be generalized to Indonesian children living in different socio-demographic and economic characteristics. Finally, other potential confounding such as child's food preferences and food availability in the household were not measured in this study.

\section{Conclusion}

Among the four food avoidance scales assessed in this study, satiety responsiveness was found to be negatively correlated with the child's body weight. Along with the child's age, family income, and slowness in eating, satiety responsiveness was also a significant predictor of children's energy intake, which was significantly correlated with the child's body weight. Parents and nutrition practitioners may find that early assessment of a child's food avoidance behaviour, especially the satiety responsiveness, could be used to identify children at risk of malnutrition. Therefore, encouraging mothers to apply responsive feeding practices, such as feed the child directly, slowly, and patiently in response to child eating behaviour as well as providing nutrition education that suggests adequate food and recommended portion sizes may have an impact on improving child diet and nutrient intake. 
Table 1. Characteristic of children aged 2-6 years old in Pejagalan, North Jakarta $(n=168)$

\begin{tabular}{lc}
\hline \multicolumn{1}{c}{ Variables } & n (\%) or Median (IQR) \\
\hline Sex & \\
Boy & $106(63.1)$ \\
Girl & $62(36.9)$ \\
Age & $4(2)$ \\
2 years old & $29(17.3)$ \\
3 years old & $38(22.6)$ \\
4 years old & $41(24.4)$ \\
5 years old & $35(20.8)$ \\
6 years old & $25(14.9)$ \\
Mother Education & \\
High & $55(32.7)$ \\
Low & $113(67.3)$ \\
Mother Occupation & $112(66.7)$ \\
Unemployed & $33(19.6)$ \\
Partially employed & $23(13.7)$ \\
Fully employed & \\
Caregiver relationship with child & $153(91.1)$ \\
Mother/father & $15(8.9)$ \\
Others (Aunt, grandmother or grandfather) & \\
Family Income Level & $24(14.3)$ \\
Low (<2 million) & $104(61.9)$ \\
Middle (2 - 4.2 million) & $40(23.8)$ \\
High (>4.2 million) & $15.3(4.1)$ \\
Body weight (kg) & $1307.3(509.9)$ \\
Energy intake (kcal) & $80(47.6)$ \\
Adequate & $88(52.4)$ \\
Risk of inadequate & \\
\hline & 3.05 million $(2.2$ million) \\
\hline &
\end{tabular}

Table 2. Distribution of food avoidance behaviour score among the subjects $(\mathrm{N}=168)$

\begin{tabular}{lc}
\multicolumn{1}{c}{ Food Avoidance Scale } & Median (IQR) \\
\hline Satiety Responsiveness & $\mathbf{3 . 2}(\mathbf{1 . 2})$ \\
My child has a big appetite* & $3(2)$ \\
My child leaves food on his/her plate at the end of a meal & $3(2)$ \\
My child gets full before his/her meal is finished & $3(2)$ \\
My child gets full up easily & $4(1)$ \\
My child cannot eat a meal if s/he has had a snack just before & $4(2)$ \\
\hline Slowness in Eating & $\mathbf{2 . 6 ( 2 . 4 )}$ \\
My child finishes his/her meal quickly* & $3(2)$ \\
My child eats slowly & $3(3)$ \\
My child eats more and more slowly during the course of a meal & $3(3)$ \\
\hline Emotional Under-eating & $\mathbf{3 . 3 ( 1 . 7 )}$ \\
My child eats less when angry & $3.5(3)$ \\
My child eats less when s/he is tired & $3(2)$ \\
My child eats less when upset & $4(2)$ \\
\hline
\end{tabular}


Table 2. Distribution of food avoidance behaviour score among the subjects (continued)

\begin{tabular}{lc}
\hline Food Avoidance Scale & Median (IQR) \\
\hline Food Fussiness & $\mathbf{2 . 7 5 ( 1 . 5 )}$ \\
My child refuses new foods at first & $3(3)$ \\
My child enjoys tasting new foods* & $3(2)$ \\
My child is interested in tasting food s/he hasn't tasted before* & $3(2)$ \\
My child decides that s/he doesn't like a food, even without tasting it & $3(2)$ \\
\hline
\end{tabular}

Scoring of the child eating behaviour questionnaire $(\mathrm{Never}=1$; Rarely $=2$; Sometime $=3$; Often $=4$; Always $=5)$

*Reversed score

Table 3. Correlation between food avoidance scales with child's weight and energy intake

\begin{tabular}{lcccccc}
\hline \multicolumn{1}{c}{ Variables } & $\begin{array}{c}\text { Body } \\
\text { weight }\end{array}$ & $\begin{array}{c}\text { Energy } \\
\text { intake }\end{array}$ & SR & SE & EUE & FF \\
\hline Body weight (kg) & 1 & & & & & \\
Energy intake (kcal) & $0.354^{* *}$ & 1 & & & & \\
Satiety responsiveness (SR) & $-0.166^{*}$ & $-0.210^{*}$ & 1 & & & \\
Slowness in eating (SE) & -0.096 & -0.037 & $0.615^{* *}$ & 1 & 1 & 1 \\
Emotional under-eating (EUE) & -0.056 & -0.048 & $0.466^{* *}$ & $0.390^{* *}$ & 1 \\
Food fussiness (FF) & 0.008 & -0.105 & $0.200^{* *}$ & 0.144 & 0.057 & \\
\hline
\end{tabular}

*Statistical analysis used Spearman correlation test with significance level of $\mathrm{P}$-value $<0.05$

${ }^{* *}$ Statistical analysis used Spearman correlation test with significance level of $\mathrm{P}$-value $<0.01$

SR: Satiety responsiveness, SE: Slowness in eating, EUE: Emotional under-eating and FF: Food fussiness

Table 4. Multiple linear regression analysis for CEBQ subscale on child's weight and energy intake

\begin{tabular}{lcccccc}
\hline \multirow{2}{*}{\multicolumn{1}{c}{ Variable }} & \multicolumn{3}{c}{ Child's weight $^{\mathbf{e}}$} & \multicolumn{3}{c}{ Energy intake $^{\mathbf{f}}$} \\
\cline { 2 - 7 } & B & SE & P value & B & SE & P value \\
\hline Child's age $^{*}$ & 1.370 & 0.158 & $<0.001^{* *}$ & 0.715 & 0.274 & $0.010^{*}$ \\
Child's sex $^{\mathrm{a}}$ & -0.186 & 0.412 & 0.652 & -0.905 & 0.715 & 0.207 \\
Mother education $^{\mathrm{b}}$ & 0.181 & 0.449 & 0.687 & 1.043 & 0.779 & 0.182 \\
Mother occupation $^{\mathrm{c}}$ & 0.332 & 0.323 & 0.306 & -0.910 & 0.561 & 0.106 \\
Caregiver relationship $^{\mathrm{d}}$ & -0.719 & 0.784 & 0.361 & 0.622 & 1.361 & 0.648 \\
Family Income $_{\text {Satiety responsiveness }}$ & 0.000 & 0.000 & 0.676 & 0.000 & 0.000 & $0.006^{* *}$ \\
Slowness in eating & -0.325 & 0.343 & 0.345 & -1.736 & 0.595 & $0.004^{* *}$ \\
Emotional under-eating & -0.048 & 0.212 & 0.823 & 0.713 & 0.368 & 0.055 \\
Food fussiness & -0.044 & 0.223 & 0.845 & 0.212 & 0.387 & 0.584 \\
\hline
\end{tabular}

Unstandardised regression coefficients (B) are from multiple linear regression models with enter method.

${ }^{*}$ Significance level at $\mathrm{P}$-value $<0.05$

** Significance level at $\mathrm{P}$-value $<0.01$

${ }^{\text {a }}$ Child's sex ( 1 = boys; 2 = girls $)$

${ }^{\mathrm{b}}$ Mother education $(1=$ high education; 2 = low education $)$

${ }^{\mathrm{c}}$ Mother occupation $(1=$ unemployed; 2 = partially employed; 3 = fully employed $)$

${ }^{\mathrm{d}}$ Caregiver relationship ( $1=$ mother/father; 2 = others (aunt, grandmother or grandfather)

${ }^{\mathrm{e}} \mathrm{R}$ square $=0.373$

${ }^{\mathrm{f}} \mathrm{R}$ square $=0.153$ 


\section{Conflict of Interest}

The authors declared no conflict of interest regarding this article.

\section{Acknowledgment}

The authors are very grateful to those who have helped and contributed to this research activity, especially Pejagalan Primary Health Care, Posyandu cadres, and all participants of this study.

\section{Open Access}

This article is distributed under the terms of the Creative Commons Attribution 4.0 International Licence

(http://creativecommons.org/licenses/by/4.0/), which permits unrestricted use, distribution, and reproduction in any medium, provided you give appropriate credit to the original author(s) and the source, provide a link to the Creative Commons license, and indicate if changes were made.

\section{References}

1. Kementerian Kesehatan Republik Indonesia. Profil Kesehatan Indonesia Tahun 2018. Jakarta: Kementerian Kesehatan RI; 2018.

2. Pasricha SR, Biggs BA. Undernutrition among children in South and South-East Asia. J Paediatr Child Health. 2010;46:497-503.

3. NB T, GA B, SM A, ME Y. Prevalence and Major Contributors of Child Malnutrition in Developing Countries: Systematic Review and Meta-Analysis. J Child Obes. 2017;2(4):16.

4. Sandjaja S, Budiman B, Harahap H, Ernawati F, Soekatri M, Widodo Y, et al. Food consumption and nutritional and biochemical status of 0.5-12-year-old Indonesian children: The SEANUTS study. Br J Nutr. 2013;110:11-20.

5. Poh BK, Ng BK, Siti Haslinda MD, Nik Shanita S, Wong JE, Budin SB, et al. Nutritional status and dietary intakes of children aged 6 months to 12 years: Findings of the Nutrition Survey of Malaysian Children (SEANUTS Malaysia). Br J Nutr. 2013;110:211-35.

6. Serra-Majem L, Ribas L, Pérez-Rodrigo C, GarcíaClosas R, Peña-Quintana L, Aranceta J. Determinants of nutrient intake among children and adolescents: Results from the enKid study. Ann Nutr Metab. 2002;46:31-8.

7. Rodenburg G, Kremers SPJ, Oenema A, van de Mheen D. Associations of Children's Appetitive Traits with
Weight and Dietary Behaviours in the Context of General Parenting. PLoS One. 2012;7(12).

8. Al-Shookri A, Al-Shukaily L, Hassan F, Al-Sheraji S, Al-Tobi S. Effect of mothers nutritional knowledge and attitudes on Omani children's dietary intake. Oman Med J. 2011;26:253-7.

9. Scaglioni S, De Cosmi V, Ciappolino V, Parazzini F, Brambilla P, Agostoni C. Factors influencing children's eating behaviours. Nutrients. 2018;10(6):1-17.

10. Shariff ZM, Lin KG, Sariman S, Lee HS, Siew CY, Yusof BNM, et al. The relationship between household income and dietary intakes of 1-10 year old urban Malaysian. Nutr Res Pract. 2015;9(3):278-87.

11. Shloim N, Edelson LR, Martin N, Hetherington MM. Parenting styles, feeding styles, feeding practices, and weight status in 4-12 year-old children: A systematic review of the literature. Front Psychol. 2015;6(1849).

12. Webber L, Cooke L, Hill C, Wardle J. Associations between children's appetitive traits and maternal feeding practices. J Am Diet Assoc [Internet]. 2010;110:1718-22. Available from: http://dx.doi.org/10.1016/j.jada.2010.08.007

13. Harbron J, Booley S, B N, CE D. Responsive feeding: establishing healthy eating behaviour early on in life. South African J Clin Nutr. 2013;26(3):S141-9.

14. Oliveira A, Jones L, De Lauzon-Guillain B, Emmett P, Moreira P, Charles MA, et al. Early problematic eating behaviours are associated with lower fruit and vegetable intake and less dietary variety at 4-5 years of age. A prospective analysis of three European birth cohorts. Br J Nutr. 2015;114:763-71.

15. Wardle J, Guthrie CA, Sanderson S, Rapoport L. Development of the children's eating behaviour questionnaire. J Child Psychol Psychiatry Allied Discip. 2001;42(7):963-70.

16. Syrad H, Johnson L, Wardle J, Llewellyn CH. Appetitive traits and food intake patterns in early life. Am J Clin Nutr. 2016;103:231-5.

17. Carnell S, Pryor K, Mais LA, Warkentin S, Benson L, Cheng R. Lunch-time food choices in preschoolers: Relationships between absolute and relative intakes of different food categories, and appetitive characteristics and weight. Physiol Behav. 2016;162:151-60.

18. Webber L, Hill C, Saxton J, Van Jaarsveld CHM, Wardle J. Eating behaviour and weight in children. Int J Obes. 2009;33:21-8.

19. Mallan KM, Nambiar S, Magarey AM, Daniels LA. Satiety responsiveness in toddlerhood predicts energy intake and weight status at four years of age. Appetite. 2014;74:79-85.

20. Gibson RS. Principles of Nutritional Assessment Rosalind S. Gibson - Google Books. Oxford University Press. 2005.

21. Börnhorst C, Huybrechts I, Ahrens W, Eiben G, Michels N, Pala V, et al. Prevalence and determinants of misreporting among European children in proxyreported $24 \mathrm{~h}$ dietary recalls. Br J Nutr. 2013;109:125765.

22. Rosidi A, Bening S, Sulistyowati E, Hunandar C, 
Wijaningsih W, Sunarto. Inadequate Energy Intake As A Risk Factor To Developmental Delay Of Pre-School Aged Children. Curr Nutr Food Sci. 2018;15:1-5.

23. Rydén PJ, Hagfors L. Diet cost, diet quality and socioeconomic position: How are they related and what contributes to differences in diet costs? Public Health Nutr. 2011;14(9):1680-92.

24. French SA, Epstein LH, Jeffery RW, Blundell JE, Wardle J. Eating behavior dimensions. Associations with energy intake and body weight. A review. Appetite. 2012;59(2):541-9.

25. Fildes A, Mallan KM, Cooke L, van Jaarsveld CHM, Llewellyn CH, Fisher A, et al. The relationship between appetite and food preferences in British and Australian children. Int J Behav Nutr Phys Act. 2015;12:116.

26. Jalkanen H, Lindi V, Schwab U, Kiiskinen S, Venäläinen T, Karhunen L, et al. Eating behaviour is associated with eating frequency and food consumption in 6-8 year-old children: The Physical Activity and Nutrition in Children (PANIC) study. Appetite. 2017;114:28-37.

27. Scaglioni S, Arrizza C, Vecchi F, Tedeschi S. Determinants of children's eating behavior. Am J Clin Nutr. 2011;94(suppl):2006S-2011S.

28. Wood AC. Appetitive Traits: Genetic Contributions to Pediatric Eating Behaviors. In: Julie C. Lumeng JOF, editor. Pediatric Food Preferences and Eating Behaviors. Academic Press; 2018. p. 127-46.

29. Carnell S, Benson L, Pryor K, Driggin E. Appetitive traits from infancy to adolescence: Using behavioral and neural measures to investigate obesity risk. Physiol Behav. 2013;10(121):79-88.

30. Carnell S, Wardle J. Appetite and adiposity in children: Evidence for a behavioral susceptibility theory of obesity. Am J Clin Nutr. 2008;88:22-9.

31. Smith AD, Herle M, Fildes A, Cooke L, Steinsbekk S, Llewellyn $\mathrm{CH}$. Food fussiness and food neophobia share a common etiology in early childhood. J Child Psychol Psychiatry. 2017;58(2):189-96. 